\title{
GERAÇÃO DE GÁS DE PIRÓLISE PARA FINS ENERGÉTICOS*
}

Adriana de Oliveira Vilela ${ }^{1}$ Ricardo Antônio Vicintin ${ }^{2}$ Thalis Pacceli da Silva ${ }^{3}$

\section{Resumo}

A produção de carvão vegetal em Forno Container Rima (FCR) consiste em um processo semi-contínuo composto por fornos cilíndricos metálicos móveis, com exaustão forçada dos gases. Cada ciclo se divide em 4 fases: carregamento, carbonização, resfriamento e descarregamento. Após o carregamento com toletes de madeira, $20 \%$ de umidade, o forno é deslocado para plataforma de carbonização, onde permanece por 3 a 4 horas. A entrada de oxigênio no interior do forno é regulada para controle das reações de pirólise. Todos os gases gerados são conduzidos a um sistema de queima para aproveitamento da energia e eliminação do metano. O peso, a temperatura e a pressão no interior do forno, bem como a vazão, composição e energia do fluxo de gás, são monitorados continuamente, em tempo real. A carbonização finaliza quando é atingido o rendimento gravimétrico de $35 \%$ em carvão e $60 \%$ em gás. 0 carvão ainda quente é transferido para cilindros de resfriamento, permanecendo por 12 horas. O forno então, é novamente carregado e se inicia um novo ciclo. Cada plataforma de carbonização pode gerar $1 \mathrm{MWe}$ de potência elétrica, produzir 20t/mês de alcatrão, 450t/mês de carvão vegetal e eliminar 500t/mês de CO2 equivalente. Cada FCR substitui 60 fornos Rabo Quente, reduzindo em mais de 50\% a mão-de-obra braçal dos processos tradicionais, pela valorização, humanização e qualificação deste trabalho. 0 carvão produzido atende aos quesitos necessários para uso como redutor na metalurgia e na siderurgia.

Palavras-chave: Carvão vegetal; Carbonização; Alcatrão; Energia elétrica.

\section{Abstract}

\section{PYROLYSIS GAS GENERATION FOR ENERGETIC PURPOSES}

The Charcoal production in Rima's Container Furnace (FCR) consists of a semicontinuous process using cylindrical furnaces with forced exhaust of the gases. Each cycle is divided into four phases: loading, carbonization, cooling and discharging. After the charging with wooden pegs, "toletes de madeira", which contains $20 \%$ moisture, the furnace is then moved to the carbonization platform where it stays for 3 to 4 hours. The introduction of oxygen into the furnace is regulated to control the pyrolysis reaction. All of the resultant gases are redirected to a heat recovery system with a secondary burner which eliminates the methane. A real time monitoring system continuously measures the weight, temperature, pressure, chemical composition and flow of materials in the furnace. The carbonization finishes when the composition of the gravimetric yield reaches $35 \%$ charcoal and $60 \%$ gas. The still warm charcoal is transferred to cooling cylinders where it stays for 12 hours. The furnace is then loaded again and a new cycle starts. Each carbonization platform can generate $1 \mathrm{MW}$ of electrical power, produce $20 \mathrm{Mt} / \mathrm{month}$ of tar, $450 \mathrm{Mt} / \mathrm{month}$ of charcoal and eliminate $500 \mathrm{Mt} / \mathrm{month}$ of $\mathrm{CO}_{2}$ equivalent. Each FCR can do the work of up to 60 conventional charcoal furnaces "raboquente", reducing the amount of manual labor required by 50\% through appreciation and qualification of this job. The charcoal produced meets the required specifications as a reduction agente in the metallurgical and steel industry.

Keywords: Charcoal; Pyrolysis gas; Tar; Electric energy.

Engenheira Química, PhD, Gerente de Pesquisa e Desenvolvimento, Departamento de Pesquisa, UFMG, Rima Industrial S/A, Belo Horizonte, Minas Gerais, Brasil.

Engenheiro Metalurgista, C\&O, Presidência, Rima Industrial S/A, Belo Horizonte, Minas Gerais, Brasil.

Engenheiro Mecânico, Chefe de Pesquisa e Desenvolvimento, Departamento de Pesquisa, CEFET, Rima Industrial S/A, Belo Horizonte, Minas Gerais, Brasil. 


\section{INTRODUÇÃO}

No Brasil, mesmo com a industrialização e a introdução de combustíveis fósseis, a lenha e o carvão vegetal de origem nativa e plantada continuam sendo fontes importantes de energia nas residências e no setor produtivo, representando $9,5 \%$ do total da oferta de energia no país, pouco menos que a oferta de energia hidráulica, que é de $13 \%$ [8]. De toda a energia consumida, $44 \%$ consiste em energia renovável, sendo 33\% energia hidráulica, e 58\% energia de biomassa [5].

O setor industrial no Brasil consumiu 6,6 milhões de toneladas de carvão vegetal em $2013,86 \%$ do consumo brasileiro. As atividades industriais que mais consumiram carvão foram: ferro-gusa (82\%), ferro-liga (13\%) e cimento (3\%) [8]. O principal estado produtor é Minas Gerais, com uma produção de 4,5 milhões de toneladas em 2012, representando $71 \%$ da produção do país. Quando se considera somente 0 carvão da silvicultura, a participação do Estado passa para 81\% [5]. Na siderurgia brasileira, cerca de $11 \%$ do aço é produzido com carvão vegetal, contribuindo de forma significativa para redução da emissão de GEE (Gases de Efeito Estufa) na atmosfera. Este setor é responsável por 16\% dessas emissões [7].

Apesar do consumo de carbono pelas florestas plantadas, durante a transformação da lenha em carvão vegetal, pelos processos tradicionais, ocorre uma emissão de $\mathrm{CO}_{2}$ equivalente à geração de $\mathrm{CH}_{4}$, da ordem de 1t de $\mathrm{CO}_{2}$ por $\mathrm{t}$ de carvão, ou seja, para uma produção nacional de 10 milhões de toneladas ano de carvão, emite-se a mesma quantidade de $\mathrm{CO}_{2}$ na atmosfera [2]; o que pode representar até $3 \%$ do total de emissões da matriz energética brasileira [6].

Segundo o Serviço Florestal Brasileiro, as florestas nativas brasileiras cobrem em torno de 516 milhões de hectares e correspondem a $60 \%$ do território nacional, sendo o $2^{\circ}$ país em área de florestas nativas [1]. As florestas energéticas que cultivam o Eucalyptus têm atingido valores recordes de produtividade mundial, até $60 \mathrm{~m}^{3} /$ há [9].

Segundo o Balanço Energético Nacional, ano base 2013, em São Paulo, dos 18.149 MW de geração elétrica instalados, em torno de 6.000MW são derivados das usinas de cana de açúcar [8].

Em Minas Gerais, dos 14.254 MW de potência elétrica instalada, a contribuição das termoelétricas é de apenas 14\%, sendo praticamente nula a participação do setor de produção de carvão vegetal [1]. Isso ocorre, porque não há no estado nenhuma tecnologia industrial consolidada que utilize os gases e derivados do processo de carbonização para geração de energia elétrica [3]. O aproveitamento da energia da carbonização em Minas Gerais, têm potencial para suprir até $10 \%$ de toda a geração de energia elétrica do estado [11].

Visando melhorias no processo de carbonização, algumas tecnologias vêm sendo desenvolvidas, em especial, as retortas verticais, horizontais e o Forno Container Rima [10]; objeto deste trabalho.

Dessa forma, o objetivo deste trabalho consistiu no desenvolvimento e projeto de um forno piloto para produção de carvão vegetal, com rendimento gravimétrico, produtividade, eficiência energética e qualidade superior aos fornos de alvenaria, o suficiente para garantir viabilidade técnica, econômica e ambiental na implantação de uma unidade de carbonização mecanizada e automatizada, associada à cogeração [10]. 


\section{MATERIAIS E MÉTODOS}

Durante a realização desta pesquisa foi utilizado o Forno Container Rima (modelo piloto), procedente da evolução dos modelos de laboratório,com embasamento em testes realizados em mufla. O Forno Container Rima (FCR) teve como origem um protótipo de menor capacidade volumétrica, tendo como objetivos principais o aumento na produtividade, na eficiência e na qualidade do carvão produzido.

O FCR foi inteiramente instrumentado, com sistemas e equipamentos periféricos, utilizados para o monitoramento contínuo e em tempo real do processo de carbonização.

Com intuito de caracterizar o processo quanto à fluidodinâmica, cinética e transferência de calor, foi utilizado o método de volumes finitos, através de softwares para modelagem e definição geométrica do forno, além de um sistema de instrumentação integrado, contendo: medidor de pressão tipo Pitot, anemômetro, termopares tipo K, sensor de temperatura por infravermelho, analisador de gases Gasboard, balanças de precisão, célula de carga, sensores e analisadores de gás, entre outros.

O desenvolvimento do projeto, contou com a seguinte metodologia cronológica:

- definição do volume de controle do estudo

- pesquisa bibliográfica e análise do estado da arte referente à pirólise lenta e geração de energia elétrica a partir de biomassa, alcatrão e gás combustível

- condução de testes para cálculos de balanço de massa e energia programáveis em planilha Excel

- análises estatísticas, utilizando os aplicativos: Minitab,Matlabe Scilab

- elaboração do balanço de massa da carbonização

- elaboração de balanços térmicos e termodinâmicos

- definição da energia de ativação global equivalente

- verificação e análise de correlação entre a taxa de decomposição térmica e a respectiva taxa de aquecimento

- análise energética do processo de carbonização em Forno Container

- proposição de métodos para homogeneização dos gradientes térmicos: longitudinal e radial no interior do forno,

- proposição de métodos para controle na geração de energia útil contida nos produtos da pirólise,

- desenvolvimento, projeto e fabricação de um forno de pirólise piloto

- definição de hipóteses, condução de testes piloto, análise dos resultados, conclusões,

- definições e validação do processo e projeto até que o mesmo apresente viabilidade técnica e econômica superiores ao forno de alvenaria;

- definição das condições ótimas de eficiência energética versus produtividade e custo na produção de carvão vegetal associado à geração de energia elétrica.

A Figura (1) representa a Forno Container versão piloto com a montagem da instrumentação para análise contínua dos parâmetros do processo, bem como da qualidade e potencial energético dos produtos gerados. 


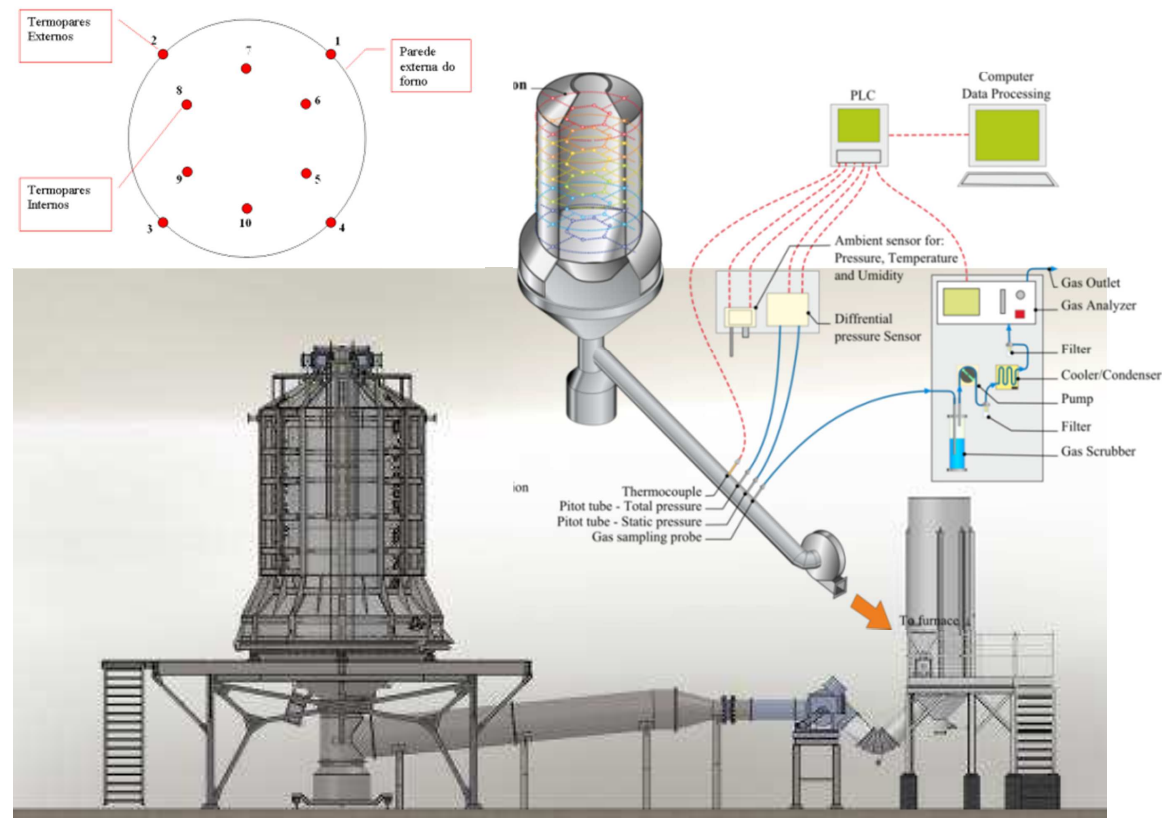

Figura 1. Sistema de Instrumentação utilizado no FCR

\section{RESULTADOS E DISCUSSÃO}

\subsection{Análise de Volumes Finitos para Modelagem do FCR}

Para definição e detalhamento do projeto do Forno Container Rima modelo piloto, foram consideradas as relações geométricas do forno laboratório, cujo modelo atingiu máxima eficiência na conversão de lenha para carvão. Relações como diâmetro/altura, volume/área, vazão de gás, quantidade, posição e abertura das válvulas de controle da entrada de ar, entre outros. A partir da conservação destes parâmetros de fabricação, o FCR foi testado, tendo uma capacidade de enfornamento de lenha de $40 \mathrm{~m}^{3}$; enquanto o forno laboratório, possuía $5 \mathrm{~m}^{3}$ de volume útil.

Com intuito de visualizar as zonas de refluxo, bolsões de gás em turbulência, ou regiões de engaiolamento com formação de buracos ou vazios, foi realizado um mapeamento fluidodinâmico do FCR, por meio de análise computacional de volumes finitos, conforme pode ser visualizado na Figura 2. Este estudo partiu das seguintes premissas: forno vazio, com condições de contorno nas válvulas: abertas, pressão atmosférica e vazão de $4.000 \mathrm{~m}^{3} / \mathrm{h}$.

As análises teóricas demonstraram a presença de formação de vórtexcom regiões de estagnação, portanto potencialmente perigosas, em função da possibilidade de acúmulo de gases combustíveis.

Para minimizar a possibilidade de ocorrência de combustão ou explosão do gás confinado no interior do forno, nas zonas de turbulência ou refluxo, foram realizadas modificações estruturais na região da base do forno, onde ocorre à saída do fluxo de gás. Além desta interferência física, foram definidos procedimentos operacionais e mudanças no processo, que corroboraram para evitar este fenômeno. 


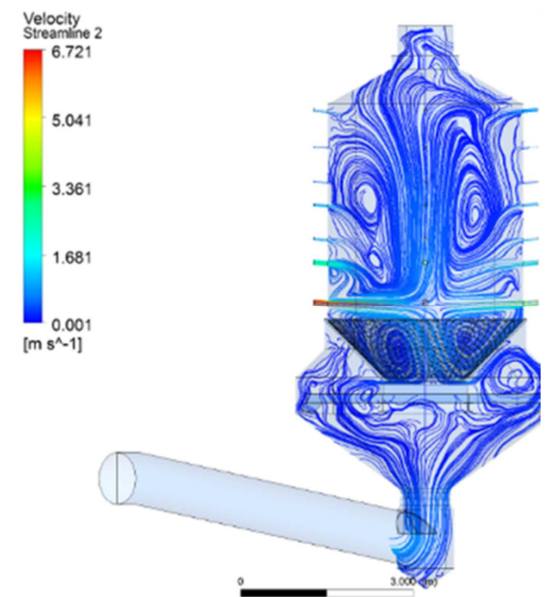

Figura 2. Simulação dos fluxos de gás no FCR vazio.

\subsection{Estudo do Perfil Térmico do FCR}

Através do sistema de monitoramento das temperaturas internas do FCR, foi possível a obtenção do perfil térmico do forno ao longo do tempo. Esse sistema é composto por 32 pontos de medição de temperatura na parede externa do forno e 42 pontos na parte interna.

Após a condução de 10 corridas de testes com o forno utilizando a parte superior para realizar a ignição, foi possível observar um comportamento padrão apresentado na Figura 3, que apresenta as temperaturas médias ao longo do tempo e ao longo da altura do forno. O nível A representa o topo do forno seguindo a sequência até a base do forno, representada pelo nível $\mathrm{H}$. Nesta figura, a linha $\mathrm{A}$ (em vermelho) representa a temperatura média, no nível $A$, ao longo do tempo das corridas nos testes realizados.

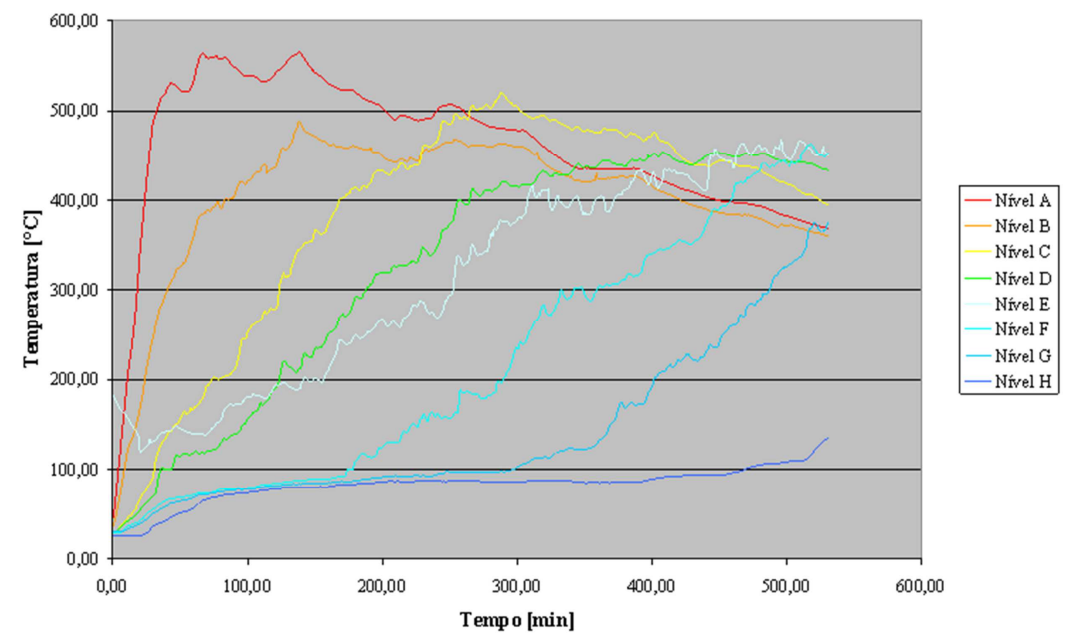

Figura 3. Gráfico de distribuição das temperaturas do FCR com ignição pelo topo

Dentre os problemas observados estão, elevada heterogeneidade térmica, menor produtividade, maior geração de tiço, entre outros. Para resolver estas questões, foram realizados novos testes, com ignição pela base. Os resultados superaram as expectativas e podem ser visualizados na Figura 4. 

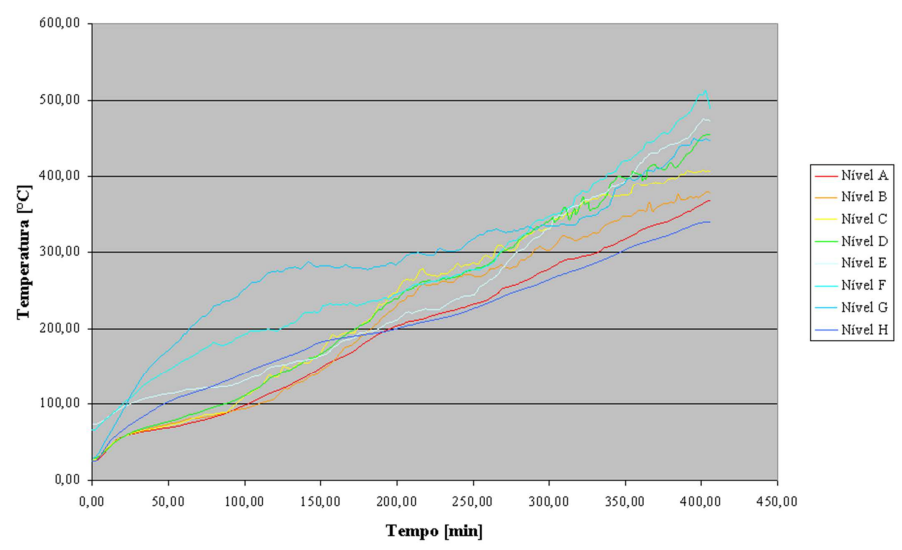

Figura 4. Gráfico de distribuição das temperaturas do FCR com ignição pela base

Com a ignição do forno sendo realizada pela base foi obtida uma série de vantagens com relação à ignição pelo topo do forno. A primeira delas é a homogeneidade da distribuição nas temperaturas, a diferença máxima entre o topo e a base do forno não ultrapassando $250^{\circ} \mathrm{C}$, garantido que o material no interior do forno percorra todas as fases da carbonização ao mesmo tempo. Esta uniformidade térmica do forno permitiu redução no tempo de carbonização para, em média, 4 horas em um forno com $40 \mathrm{~m}^{3}$ de capacidade. Outra vantagem para este sistema é o aumento nas temperaturas da base do forno, permitindo a carbonização completa de toda a lenha.

\subsection{Estudo da Cinética do FCR}

Para os cálculos dos balanços de massa e energia foi necessário conhecer a energia de ativação da reação de pirólise. Como o processo de carbonização não ocorre em uma única temperatura, o estudo da cinética no processo em escala piloto, foi realizado a partir de dados termogravimétricos reais. Dessa forma, foram monitorados 30 testes de carbonização, tendo sido obtida a taxa de decomposição da madeira em quilos/minuto e as curvas de temperatura da madeira e carvão ao longo do ciclo.A energia de ativação foi calculada pela equação de Arrhenius.

Neste trabalho esta função será expressa conforme abaixo [4]:

$\frac{d x}{d t}=A \cdot \exp \left(-\frac{E}{R} \cdot T\right) \cdot(1-x)$

A: fator pré-exponencial

E: energia de ativação

$\mathrm{X}$ : taxa de conversão da madeira em carvão

$\mathrm{R}: 8,314 \mathrm{~kJ} / \mathrm{kmol} . \mathrm{K}$

$\mathrm{X}=$ taxa de perda de peso

$\beta=\mathrm{dT}$ (taxa de aquecimento constante)

$\mathrm{dt}$

Tendo sido gerados estes valores, foram plotadas as curvas de $\ln [-\ln (1-x)] / T^{2}$ versus $1 / T$. Foi calculada a Energia de Ativação $(E)$, que corresponde à inclinação da curva e com este valor, pode-se calcular o fator pré-exponensial A.

Considerando a madeira com um PM em torno de $188 \mathrm{~g} / \mathrm{mol}$; então a energia de ativação da pirólise no FCR foi de $74 \mathrm{~kJ} / \mathrm{kg}$. 


\subsection{Balanço de Massa e Energia da Carbonização no FCR}

Para o estudo dos balanços de massa e energia do processo de carbonização no FCR, foram realizadas 10 corridas, com monitoramento de todos os parâmetros necessários, como perda de peso e perfil térmico volumétrico da carga de lenha, além de vazão, composição química, pressão e temperatura dos gases não condensáveis, bem como produção de carvão, alcatrão e pirolenhoso.

A análise desses dados culminou na curva de potência térmica do fluxo de gases não condensáveis gerados, conforme apresentado na Figura 5.

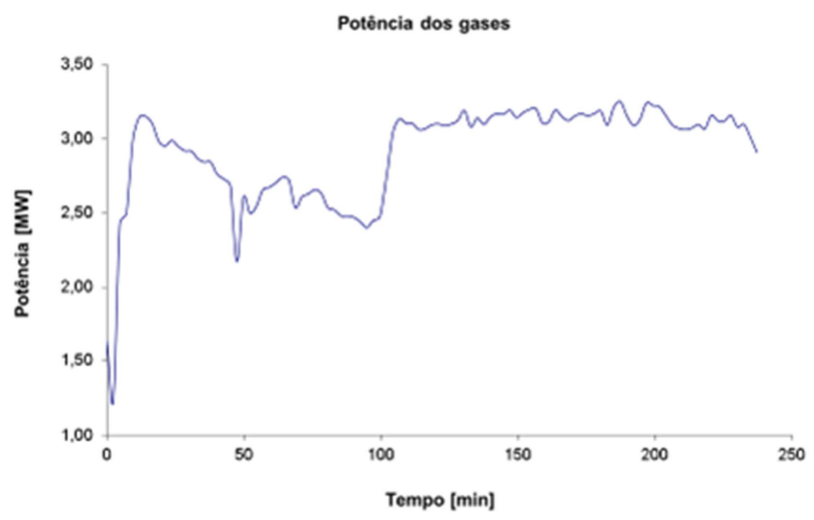

Figura 5. Gráfico de Potência dos Gases por Forno

Para confirmação deste resultado, foram calculadas as energias utilizadas em cada etapa do processo: secagem, pré-pirólise e pirólise, considerando todas as energias envolvidas, como perdas térmicas por condução e convecção, calor sensível, calor latente, entalpias, energia de ativação, energia disponível, além de percentual de oxidação dos compostos combustíveis (carvão, lenha, gás, alcatrão), percentual de combustão completa e excesso de oxigênio nas reações. Para fechamento dos balanços de massa e energia foi utilizado o software Solver, que partiu da premissa de que 0 erro decorrente da diferença entre a composição química dos gases medida e a composição calculada pelas equações termodinâmicas deveria ser mínimo. Os resultados demonstraram um erro inferior a 2\%,conformeTabela 1.

Tabela 1. Resultados obtidos pela ferramenta SOLVER, na redução do erro global para o balanço de massa e energia.

\begin{tabular}{|l|c|}
\hline \%Excesso & $0 \%$ \\
\%Combustão Completa & $47 \%$ \\
$\%$ reação com vapor completa & $100 \%$ \\
$\%$ GNC & $84 \%$ \\
$\%$ Alcatrão $+\mathrm{O}_{2}$ & $90 \%$ \\
$\%$ Pirolenhoso $+\mathrm{O}_{2}$ & $31 \%$ \\
$\%$ Alcatrão $+\mathrm{H}_{2} \mathrm{O}$ & $0 \%$ \\
$\%$ Pirolenhoso $+\mathrm{H}_{2} \mathrm{O}$ & $54 \%$ \\
Teor de umidade no pirolenhoso & $20 \%$ \\
Premissa do BM para alcatrão & $90 \%$ \\
Premissa do BM para pirolenoso & $85 \%$ \\
Potência Térmica (MW) & $\mathbf{3 , 5}$ \\
\hline Erro Global & $\mathbf{1 , 5 0 \%}$ \\
\hline
\end{tabular}


O valor da potência térmica disponível também foi equivalente ao valor medido, conforme Tabela 2.

Tabela 2. Resultados obtidos pela ferramenta SOLVER, na redução do erro globalpara o balanço de massa e energia.

\begin{tabular}{|lcc|}
\hline ENERGIA DO GÁS & \\
MASSA TOTAL DE GÁS NA SAÍDA & $27.303 \mathrm{~kg}$ \\
PCI DO GÁS PELO SOLVER & $329 \mathrm{kCal} / \mathrm{kg}$ \\
PCI DO GÁS PELO GASBOARD & $425 \mathrm{kCal} / \mathrm{kg}$ \\
ENERGIA DO GÁS MEDIDO/Gasboard & & $3.865 .402 \mathrm{kCal} / \mathrm{h}$ \\
ENERGIA DO GÁS CALCULADO & & $2.996 .017 \mathrm{kCal} / \mathrm{h}$ \\
\hline POTÊNCIA TÉRMICA Medido/Gasboard & 4,5 & $\mathrm{MW}$ \\
DISPONÍVEL $\quad$ Calculado/Solver & 3,5 & $\mathrm{MW}$ \\
\hline
\end{tabular}

Os resultados demonstraram que a tecnologia FCR é energeticamente eficiente, com uma perda térmica inferior a $5 \%$, rendimento gravimétrico em carvão de $35 \%$ e rendimento gravimétrico em gás de $60 \%$. Estes valores estão representados na Figura 6.

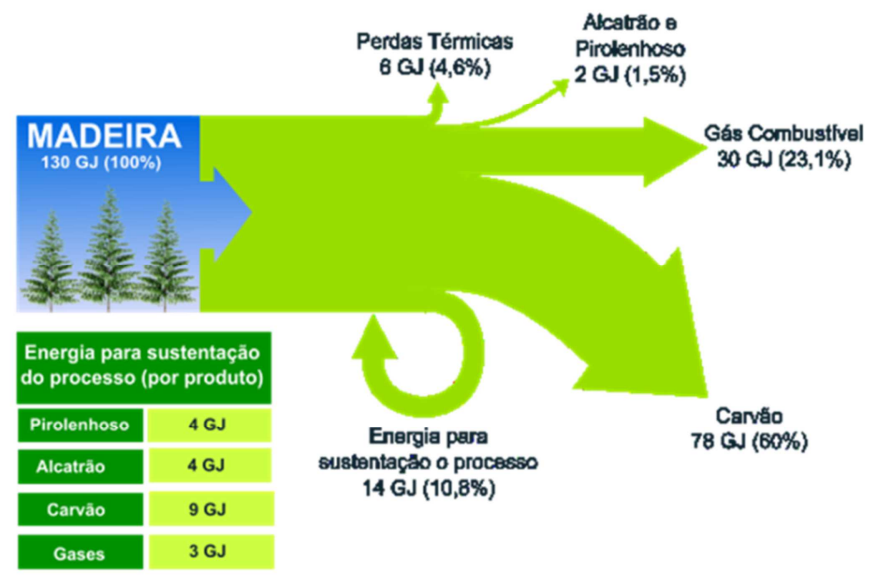

Figura 6. Balanço de Energia da Carbonização no FCR

\section{CONCLUSÃO}

O FCR atingiu um estágio tecnológico decorrente do desenvolvimento dos fornos de laboratório e piloto, com aperfeiçoamento contínuo. Os resultados demonstraram uma conversão gravimétrica em carvão vegetal, tendo como média $34 \%$ e um rendimento em gás combustível em torno de $60 \%$. Com estes valores, cada FCR tem potencial para uma geração térmica em torno de $4 \mathrm{MW}$. Considerando uma unidade de carbonização com FCR, com 8 plataformas, associada à uma planta de cogeração, pode-se gerar entre 8 e 10MW, dependendo do uso dos sub-produtos. Para uma térmica de $20 \mathrm{MW}$, uma UPC de $25.000 \mathrm{~m}^{3} / \mathrm{mês}$ de carvão, pode fornecer até $30 \%$ da energia necessária para geração de eletricidade.

O carvão vegetal produzido no FCR apresenta qualidades semelhantes ao carvão da alvenaria e adequadas ao uso como redutor nos fornos elétricos.

O Balanço de Massa e Energia demonstrou uma perda térmica no FCR de 5\% e uma energia de sustentação de $10 \%$. Portanto uma eficiência de $85 \%$. 


\section{Agradecimentos}

A Rima Industrial S/A, empresa empreendedora, responsável pelo desenvolvimento contínuo e tecnológico de uma alternativa ao processo tradicional de carbonização.

A Financiadora de Estudos e Projetos (FINEP), pelo crédito técnico e financeiro, cuja apropriação viabilizou este projeto (2010).

Ao Dr.Electo Silva Lora da Universidade Federal de Itajubá (Núcleo de Excelência em Geração Termoelétrica e Distribuída).

\section{REFERÊNCIAS}

1 AMS Associação Mineira de Silvicultura. Anuário Estatístico. 2012 [acesso em 15 fev 2015]. Disponível em: http://www.bibliotecaflorestal.ufv.br/handle/12356789/13195

2 Bailis R, Miranda RC, Vilela AO, Cogenerating Electricity From Charcoaling: A Promising New Advanced Technology. Energy for Sustainable Development. V 17. 2013. 171-176.

3 Bailis R, Miranda RC, Vilela AO, Chang H, Rujanavech C, Dwivedi P. Innovation in charcoal production: A comparative life-cycle assessment of two kiln technologies in Brazil. Energy for SustainableDevelopment. 2012 [acesso em 13 fev 2013]. Disponível em: http://dx.doi.org/10.1016/j.esd.2012.10.008

4 Himawanto Da. Thermogravimetric Analysis and Global Kinetics of Segregated MSW Pyrolysis. Modern Applied Science. Republic of Indonesia, v.6, No. 1; Jan, 2012.

5 IBGE Instituto Brasileiro de Geografia e Estatística. Produção da extração vegetal e da silvicultura. 2012 [acesso em 12 out 2013]. Disponível em: http://www.ibge.gov.br.

6 IEA International Energy Agency. Energy balances of non-oecd countries $2003-2004$. Paris: OECD, 2006. 365 p.

7 Mello AV. Siderurgia e biomassa: uma solução mais sustentável. Revista Opiniões Divisão florestal dez-fev 2015. 38. ISSN 2177-6504.14-15.

8 MME Ministério de Minas e Energia. Balanço energético Nacional 2014. 2014 [acesso em: 12 abr 2015]. Disponível em: http://ben.epe.gov.br

9 SNIF Sistema Nacional de Informações Florestais. Recursos Florestais: As Florestas Plantadas. 2015 [acesso em 14 abr. 2015]. Disponível em: http://www.florestal.gov.br/snif/recursos-florestais/as-florestas-plantadas.

10 Vilela, AO. Desenvolvimento e validação técnica, de um forno industrial de carbonização, modelo container $2^{a}$ geração. Belo Horizonte. Tese [Doutorado em Saneamento, Meio Ambiente e Recursos Hídricos] - Universidade Federal de Minas Gerais; 2013

11 Vilela AO, Vicitin RA, Souza TPS, Lora ES, Quintero QR. A New Technology for the Combined Production of Charcoal and Electricity Through Cogeneration.

Biomass\&Bioenergy. V 69. 2014. 222-240. 